\title{
ENDOMETRIOSIS PROFUNDA DEL TABIQUE RECTOVAGINAL CON COMPROMISO INTESTINAL: MANEJO QUIRÚRGICO CON RESECCIÓN SEGMENTARIA DE RECTOSIGMOIDES
}

\author{
Guillermo Durruty V. ${ }^{1}$, Demetrio Larraín de la C. ${ }^{1}$, Mauricio Cuello F. ${ }^{1}$, Cristián Po- \\ més C. ${ }^{1}$, Hugo Vidal A. ${ }^{2}$, Angie Vergara R. ${ }^{1}$, Waldo Leiva L. ${ }^{1}$
}

\footnotetext{
${ }^{1}$ Departamento de Obstetricia y Ginecología, Facultad de Medicina, Pontificia Universidad Católica de Chile. ${ }^{2}$ Médico General, Universidad Católica de Chile.
}

\section{RESUMEN}

Presentamos 3 casos de endometriosis profunda del tabique rectovaginal con compromiso intestinal, en los cuales se realizó resección segmentaria del rectosigmoides para lograr la remoción completa de la enfermedad. Se analiza el cuadro clínico, manejo quirúrgico, complicaciones y seguimiento posterior. Revisamos la literatura a fin de establecer algunas pautas de manejo de esta entidad.

\section{PALABRAS CLAVES: Endometriosis profunda, laparoscopia, nódulo rectovaginal}

\section{SUMMARY}

We reported 3 cases of deep endometriosis affecting the rectovaginal space with intestinal disease in which a rectosigmoides resection was required to achieve a complete surgical removal of disease. The clinical course, surgical management, complications and follow-up are analyzed. We review the literature to define some guidelines in the management of this entity.

\section{KEY WORDS: Deep infiltrating endometriosis, laparoscopy, rectovaginal nodule}

\section{INTRODUCCIÓN}

La endometriosis se define como la presencia de tejido endometrial funcional (glándulas y estroma) fuera de la cavidad uterina (1). Desde un punto de vista anatómico la endometriosis puede tener localización pelviana o extrapelviana. Dentro de la endometriosis pelviana se distinguen tres formas: la endometriosis ovárica, la endometriosis peritoneal superficial y la endometriosis peritoneal profunda. Esta última corresponde a la forma más rara de las tres mencionadas y se diagnostica cuando existe infiltración del espacio retroperitoneal $\geq 5 \mathrm{~mm}$
(2). Su incidencia se estima 1 en 170 a 1 en 3.800 mujeres. La endometriosis del tabique rectovaginal (TRV) corresponde a la forma más severa de la endometriosis profunda comprometiendo específicamente el tejido conectivo entre la vagina y la pared anterior del recto. Se estima que 1 de cada 6 mujeres con endometriosis profunda presentará compromiso del TRV lo que representa 1 de cada 1000 a 1 en 23.000 mujeres con endometriosis (3).

La endometriosis del TRV puede infiltrar tanto la vagina como el recto $y$, en los casos más severos, puede extenderse lateralmente llegando a comprometer uno o ambos uréteres $(3,4)$. El com- 
promiso intestinal aunque infrecuente, se observa en el $3-37 \%$ de las pacientes con endometriosis y afecta principalmente el rectosigmoides (5-9). El tratamiento médico es ineficaz y tiene escaso rol en el manejo de estas pacientes (10), las que con frecuencia requieren de un tratamiento quirúrgico agresivo, que puede incluir la resección de un segmento intestinal para lograr un tratamiento exitoso (11).

El objetivo de este trabajo es revisar la experiencia del Hospital Clínico de la Pontificia Universidad Católica de Chile en el enfrentamiento diagnóstico y terapéutico de pacientes con endometriosis profunda con compromiso intestinal (rectosigmoides). Se presenta además, una revisión de la evidencia disponible en la literatura con el fin de entregar algunas pautas para su diagnóstico y manejo correcto.

\section{PACIENTES Y MÉTODO}

Se revisaron los registros de pabellón de nuestro centro y se construyó una base de datos con los casos de endometriosis operados entre enero de 2005 y diciembre de 2007. Se reclutaron todas las pacientes con diagnóstico de endometriosis del TRV que fueron tratadas quirúrgicamente y con biopsia procesada en el Servicio de Anatomía Patológica del Hospital Clínico de la Universidad Católica de Chile. Retrospectivamente se revisaron las fichas clínicas de aquellos casos en que el manejo quirúrgico requirió de una resección colorrectal segmentaria por compromiso profundo o transmural de la pared intestinal. Fueron excluidas del análisis, aquellas pacientes en las que se evidenció la presencia de implantes serosos superficiales en el intestino y que sólo requirieron fulguración superficial del peritoneo, sin entrada al lumen intestinal, o aquellas en que se realizó una resección en cuña, sin resección intestinal segmentaria,. Se incluyeron variables clínicas, como edad al diagnóstico, motivo de consulta, antecedentes de tratamiento médico para endometriosis, de tratamiento quirúrgico por endometriosis, exámenes de evaluación preoperatoria, la vía de abordaje quirúrgico, el tipo de cirugía realizada, el tiempo operatorio, las complicaciones y seguimiento. En todos los casos incluidos hubo confirmación histológica en la pieza de compromiso intestinal profundo por endometriosis. Para el seguimiento se registraron los controles postoperatorios y se efectuó una entrevista telefónica, orientada a determinar la presencia de síntomas, embarazo, complicaciones a largo plazo y satisfacción con la cirugía.

Los antecedentes clínicos para su análisis fue- ron registrados en una base de datos Excel versión office 2007.

\section{RESULTADOS}

En el período de estudio se registraron un total de 30 casos de endometriosis profunda con compromiso del TRV. De ellos, sólo 3 casos (10\%) presentaron compromiso colorrectal con necesidad de resección segmentaria.

La edad promedio de las pacientes fue 32 años (rango: 29-33 años). En todas las pacientes existía el antecedente de al menos una cirugía por endometriosis y/o presentaba recurrencia de los síntomas pese al uso de tratamiento médico.

En los tres casos se consigna dentro los motivos de consulta disquexia catamenial, defecación dolorosa, dispareunia profunda y dismenorrea severa. Una de las pacientes refirió episodios de hematoquezia y rectorragia catamenial. Basado en dichos síntomas, en los 3 casos, hubo sospecha preoperatoria de compromiso intestinal. Al tacto vaginal, en los 3 casos se palpaba nódulos sensibles en relación al fórnix posterior. Sin embargo, en el tacto rectovaginal, no se palpaba nódulos o puntos dolorosos específicos. Cabe señalar que en una de las pacientes fue necesario realizar el examen bajo anestesia debido a la intensidad de su sintomatología.

El estudio por imágenes incluyó la resonancia magnética (RM) de pelvis y la endosonografía rectal. En 2 de los casos, la RM confirmó la presencia de un nódulo en el TRV, uno de ellos con compromiso transmural evidente del recto. Por su parte, la endosonografía fue sugerente de infiltración de la pared rectal en un caso. Dado el gran tamaño del nódulo (mayor de $3 \mathrm{~cm}$ ), en dos casos se realizó pielografía de eliminación. Ninguna de las pacientes refería síntomas urinarios irritativos u obstructivos previo a su diagnóstico. En los dos casos la pielografía mostró hidroureteronefrosis bilateral. En ambos se instaló un catéter doble $\mathrm{J}$ bilateralmente, en forma retrógrada, mediante cistoscopía, previo a la cirugía. Tal instalación se realizó con el fin de facilitar la identificación y liberación de los uréteres durante la cirugía (ureterolisis). Cabe señalar que una de estas pacientes, pese a la instalación de un doble $\mathrm{J}$ bilateral, presentó insuficiencia renal posrenal secundaria a la obstrucción ureteral, requiriendo de compensación de su función renal previo a la cirugía.

Por la sospecha clínica de compromiso intestinal, a todas las pacientes se les realizó una colonoscopia. En una de ellas (caso 3) había compromiso evidente de la mucosa rectal. En dicho caso 
se tomó biopsias por endoscopia dado su aspecto inflamatorio y necrótico, sugerente de compromiso neoplásico. Dichas biopsias sólo mostraron inflamación y fibrosis.

El tratamiento fue quirúrgico con enfoque conservador de la fertilidad (preservación del útero y al menos un ovario) en los 3 casos. En 2 de los casos el abordaje fue primariamente por vía laparoscópica. En el tercer caso se optó por la laparotomía dado el compromiso ureteral bilateral y la complejidad de la cirugía.

Independiente de la vía de abordaje, en todas las pacientes se realizó preparación mecánica del intestino y profilaxis antibiótica según normativa local (12). La cirugía fue planificada y realizada por un equipo multidisciplinario compuesto por ginecólogo, coloproctólogo y urólogo.

Una vez realizado el pneumoperitoneo e instalada la óptica (o abierta la cavidad abdominal), en las 3 pacientes, el hallazgo principal fue la presencia de sellamiento u obliteración del fondo de saco posterior. Liberada la pelvis fue posible demostrar la presencia de endometriosis profunda con compromiso del TRV en los tres casos. La coexistencia de endometriosis ovárica se observó sólo en una paciente. En dos pacientes se evidenció la extensión lateral del nódulo. Abierto el peritoneo retrouterino y desarrollados los espacios avasculares de la pelvis (espacios pararrectales) se demostró el compromiso uni o bilateral del uréter en dos casos.

En las tres pacientes, a fin de lograr la resección completa del nódulo TRV, se realizó resección segmentaria del rectosigmoides con anastomosis primaria utilizando corchetera. La evaluación del adecuado sello de la anastomosis se realizó mediante una prueba neumática insuflando aire transrectal (sonda Petzer). En una de las pacientes, dada la magnitud de la cirugía (anastomosis primaria ultra baja) se realizó una ileostomía de protección la cual se cerró en forma diferida 3 meses más tarde.

En una de las pacientes abordadas primariamente por laparoscopia fue necesaria la conversión. Dicha conversión se debió a la necesidad de realizar un reimplante ureteral. Tal procedimiento se realizó en los 2 casos con compromiso ureteral. En uno de ellas la técnica de reimplante requirió de una vejiga psoica. Dicha paciente presentaba extensión lateral del nódulo con compromiso ureteral unilateral. En la otra paciente, con un compromiso extenso y bilateral de los uréteres (misma paciente que experimentó insuficiencia renal) fue necesaria la remoción segmentaria de ambos uréteres en la porción adherida al nódulo. Dada la longitud del segmento resecado, el reimplante se hizo mediante la técnica de colgajo vesical de Casati-Boari.
En una de las tres cirugías fue posible realizar el procedimiento completo por vía laparoscópica, remoción completa del nódulo, la resección intestinal y anastomosis primaria.

El tiempo operatorio promedio fue de $403 \mathrm{mi}-$ nutos, siendo mayor en los casos con reimplante ureteral.

En todos los casos hubo confirmación histológica de endometriosis profunda del TVR con compromiso de la pared colorrectal (la Figura 1 ilustra uno de los casos).

En el período postoperatorio inmediato no se registraron complicaciones mayores (incluyendo filtración de la anastomosis). Uno de los casos requi-

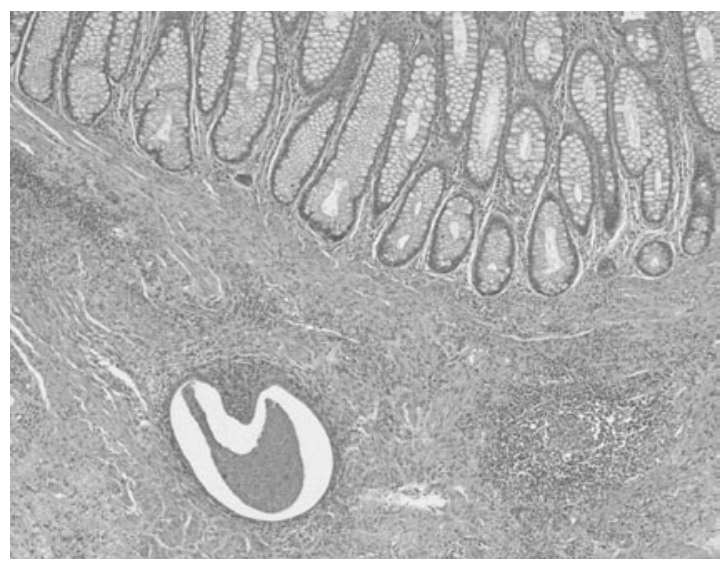

Figura 1. Microfotografía de pieza quirúrgica de colon sigmoides en que se observa infiltración por tejido endometriósico de la pared intestinal (Tinción hematoxilina y eosina, 40X).

rió de transfusión producto de anemia sintomática. Sin embargo, hubo una complicación mayor tardía, posterior al alta de la paciente, que correspondió a un tromboembolismo pulmonar 2 semanas posterior al alta. La paciente fue rehospitalizada y se instaló un filtro en vena cava inferior más el inicio de anticoagulación (TACO), que se indicó por un mínimo de 3 meses.

Con un seguimiento promedio de 20 meses (rango: 16-26 meses), 2 de las 3 pacientes se encuentran en buenas condiciones. La tercera requirió de una reexploración producto de la estenosis del reimplante ureteral. Recientemente la paciente con el tromboembolismo pulmonar finalizó su TACO; en su control de imágenes se constata la presencia de endometriosis ovárica bilateral, motivo por el cual se ha planificado una cirugía de reexploración.

La Tabla I resume las características clínicas, los aspectos quirúrgicos y de seguimiento de las tres pacientes incluidas en la serie. 
Tabla I

RESUMEN DE LAS CARACTERÍSTICAS CLÍNICAS Y DE LABORATORIO DE LAS PACIENTES INCLUIDAS EN LA SERIE

\begin{tabular}{|c|c|c|c|}
\hline Caso & 1 & 2 & 3 \\
\hline Edad (años) & 29 & 33 & 32 \\
\hline Fórmula de paridad & GOPOAO & GOPOAO & GOPOAO \\
\hline Infertilidad & Sí & No & No \\
\hline Dispareunia & No & No & Sí \\
\hline Disquexia & Sí & Sí & Sí \\
\hline $\begin{array}{l}\text { Algia pel viana no } \\
\text { cíclica }\end{array}$ & No & Sí & Sí \\
\hline Dismenorrea & Sí & Sí & Sí \\
\hline Rectorragia cíclica & Sí & No & Sí \\
\hline Endosonografía & No realizada & $\begin{array}{r}\text { Lesión hipoecogénica } \\
\qquad 6 \times 4 \mathrm{~cm}\end{array}$ & No realizada \\
\hline RNM de pelvis & $\begin{array}{l}\text { Lesión retrouterina, } \\
\text { nódulo del TRV }\end{array}$ & No realizada & $\begin{array}{c}\text { Nódulo TRV } 5 \times 5 \mathrm{~cm} \\
\text { con compromiso rectal } \\
\text { y ureteral bilateral }\end{array}$ \\
\hline USTV & $\begin{array}{l}\text { Endometrioma } \\
\text { izquierdo }\end{array}$ & Normal & Normal \\
\hline USTR & No realizada & No realizada & No realizada \\
\hline Colonoscopia & $\begin{array}{l}\text { Comprensión extrinseca } \\
\text { pared rectal lateral }\end{array}$ & $\begin{array}{l}\text { Abombamiento cara } \\
\text { anterior del recto }\end{array}$ & $\begin{array}{r}\text { Lesiones inflamatorias } \\
\text { eritematosas }\end{array}$ \\
\hline $\begin{array}{l}\text { Pielografía de } \\
\text { eliminación }\end{array}$ & No realizada & $\begin{array}{r}\text { Hidroureteronefrosis } \\
\text { bilateral, infiltración } \\
\text { ambos uréteres }\end{array}$ & No realizada \\
\hline Tipo nódulo & ॥ & III & III \\
\hline $\begin{array}{l}\text { Cirugías previas por } \\
\text { endometriosis }\end{array}$ & 1 Laparoscopia & 2 Laparoscopias & $\begin{array}{l}1 \text { Laparoscopia, } \\
\text { nefrostomía percutánea }\end{array}$ \\
\hline $\begin{array}{l}\text { Tratamiento médico } \\
\text { previo }\end{array}$ & Agonistas $\mathrm{GnRH}, \mathrm{ACO}$ & $\begin{array}{r}\text { AINEs, ACO, } \\
\text { Agonistas GnRH }\end{array}$ & Agonistas GnRH \\
\hline Vía de abordaje & Laparoscopia & $\begin{array}{l}\text { Laparoscopia, } \\
\text { conversión a } \\
\text { laparotomía }\end{array}$ & Laparotomía \\
\hline Cirugía realizada & Resección anterior & $\begin{array}{r}\text { Resección anterior, } \\
\text { reimplante ureteral } \\
\text { unilateral }\end{array}$ & $\begin{array}{c}\text { Resección anterior } \\
\text { baja, reimplante } \\
\text { ureteral bilateral }\end{array}$ \\
\hline Endometriomas & Sí, izquierdo & No & No \\
\hline Tiempo operatorio & 370 minutos & 480 minutos & 360 minutos \\
\hline Días hospitalización & 6 & 10 & 12 \\
\hline Biopsia & $\begin{array}{r}\text { Endometriosis pared } \\
\text { rectal }\end{array}$ & $\begin{array}{r}\text { Endometriosis pared } \\
\text { rectal y uréter }\end{array}$ & $\begin{array}{l}\text { Endometriosis pared } \\
\text { rectal y ambos uréteres }\end{array}$ \\
\hline Fertilidad posterior & No & No & No (sin intento) \\
\hline Complicaciones & No & No & TVP y TEP \\
\hline Mejoría síntomas & Sí & $\begin{array}{r}\text { Parcial, se reopera por } \\
\text { dolor al año }\end{array}$ & $\begin{array}{r}\text { Parcialmente, } \\
\text { desarrolla síntomas y } \\
\text { endometriomas, al año } \\
\text { de seguimiento }\end{array}$ \\
\hline
\end{tabular}

G/P/A: gestaciones/partos/abortos. RNM: resonancia nuclear magnética. USTV: ultrasonografía transvaginal USTR: ultrasonografía transrectal. ACO: Anticonceptivos orales 


\section{DISCUSIÓN}

La endometriosis intestinal fue descrita por primera vez por Sampson en 1922 (13), y se define como la infiltración de la pared intestinal por tejido endometrial ectópico, comprometiendo la muscular propia (14). La incidencia reportada oscila entre 3 y $37 \%$ y depende del centro de donde provenga la serie (5-9). Habitualmente las mayores incidencias se observan en centros de referencia como el nuestro. En la actualidad, la incidencia estimada para nuestro centro es de un $10 \%$.

Tratar de definir un cuadro clínico característico para la endometriosis profunda con compromiso intestinal, que le distinga de otras formas de endometriosis, es difícil. Sin embargo, ciertos elementos de la anamnesis nos deben hacer pensar en ella. En primer lugar, debe tenerse presente que la endometriosis del TRV se presenta principalmente con dolor pélvico, el cual puede tener diferentes patrones (ej. dismenorrea, dispareunia profunda, algia pelviana no cíclica). Junto a este patrón de dolor "típico", pueden coexistir síntomas gastrointestinales bajos y/o cambios en el hábito intestinal, todos ellos secundarios al compromiso colorrectal, como son la rectorragia, el tenesmo rectal, la disquexia catamenial y la defecación dolorosa durante la menstruación $(8,15,16)$. Por lo tanto la asociación de dolor pélvico a alguno de estos síntomas debe motivar la búsqueda dirigida de dicho compromiso. Pero más importante aún, es entender que el cuadro clínico de la endometriosis con compromiso intestinal es muy variado, haciendo su diagnóstico difícil y requiriendo de un alto índice de sospecha. Por ende, recomendamos fuertemente que en toda paciente con diagnóstico presuntivo o conocido de endometriosis severa, se le interrogue dirigidamente en busca de alguno de estos síntomas ya que existe una correlación entre el antecedente de endometriosis, su localización y la probabilidad de compromiso intestinal $(16,17)$. A modo de ejemplo, en nuestra serie, todas las pacientes tenían el antecedente de endometriosis severa y presentaban alguno de los síntomas ya descritos.

La utilidad del examen físico en el diagnóstico de endometriosis del TRV es motivo de controversia, pese a ello no debe ser omitido. Los hallazgos son variados y dependen de la localización de las lesiones y del día del ciclo menstrual en que se realice el examen (18). Si bien lo más frecuente es palpar un nódulo sensible en el fondo de saco vaginal posterior (evocando en forma exacta la sintomatología referida por la paciente), su ausencia no le descarta. Según Chapron, en un $65 \%$ de las pacientes con endometriosis profunda y compromi- so rectal no se palpan nódulos dolorosos al tacto vaginal (19). Así también la presencia de lesiones rojas durante la especuloscopía constituye otro hallazgo altamente sugerente de endometriosis del TRV. Sin embargo, tal hallazgo está ausente en el $70 \%$ de los casos de endometriosis intestinal (19). Con respecto al tacto rectovaginal, además de ser un procedimiento molesto, es claro que su utilidad es limitada $(11,20)$, puesto que las lesiones habitualmente se ubican más altas quedando fuera del alcance. Por ello, es que no lo realizamos en forma rutinaria. En base a nuestra experiencia, recomendamos fuertemente examinar a la paciente durante el periodo menstrual, particularmente si el examen clínico inicial ha sido dudoso y la anamnesis sugiere la presencia de endometriosis profunda.

Con el fin de maximizar el éxito terapéutico, resulta medular el establecer con la mayor exactitud posible la real extensión y localización de la enfermedad. Este conocimiento influirá sobre la planificación del tratamiento (incluyendo quienes participarán de su manejo) y sobre el riesgo de recurrencia de la enfermedad producto de tratamientos parciales, llevando consigo un aumento en los costos y agregando morbilidad.

Al respecto, algunos autores han definido los exámenes que deben ser realizados ante la sospecha de esta entidad y según su resultado han establecido clasificaciones que permiten predecir la complejidad del tratamiento requerido y la posibilidad de fracaso de éste $(14,21)$. Dentro de esas clasificaciones, en nuestro centro, hemos utilizado la clasificación propuesta por Donnez y cols (22), que considera los hallazgos en la resonancia magnética $(\mathrm{RM})$ de pelvis y a la ultrasonografía transrectal (USTR) (Tabla II). Cabe señalar que en estas pacientes, es frecuente el uso de diferentes métodos de imagen en pos de aumentar la precisión diagnóstica (23). La Tabla III resume los valores de sensibilidad, especificidad, valor predictivo y likelihood ratio para los distintos métodos de imagen utilizados para el diagnóstico de endometriosis profunda con compromiso intestinal.

Dentro de los exámenes utilizados, la USTR constituye un examen útil frente a la sospecha de endometriosis del tabique rectovaginal y de compromiso intestinal. Sin embargo, para su correcta interpretación se requiere de personal entrenado y de realización rutinaria, situación que no ocurre en muchos centros (24). En nuestro centro aunque se realiza, aún no es de indicación habitual. Otros centros han preconizado el uso de la ultrasonografía transvaginal (USTV). Bajo dicha imagen, las lesiones endometriósicas aparecen como engrosamientos lineales o nódulos hipoecoicos de tamaño varia- 
Tabla II

CLASIFICACIÓN DE DONNEZ DE LOS NÓDULOS ENDOMETRIÓSICOS (ADENOMIÓTICOS) RETROPERITONEALES (22)

\begin{tabular}{|c|c|}
\hline Tipo nódulo & Características \\
\hline Tipo I & $\begin{array}{l}\text { Ubicados en tabique rectovaginal } \\
\text { Usualmente de tamaño } \leq 2 \mathrm{~cm} \\
\text { (corresponde al } 15 \% \text { de los nódulos } \\
\text { retroperitoneales) }\end{array}$ \\
\hline Tipo II & $\begin{array}{l}\text { Ubicación retrocervical (fórnix posterior) } \\
\text { (tipo más frecuente, } 60 \% \text { de los nódulos) }\end{array}$ \\
\hline Tipo III & $\begin{array}{l}\text { En forma de "reloj de arena" o Diabolo-Like } \\
\text { Usualmente de tamaño } \geq 3 \mathrm{~cm} \\
\text { Usualmente infiltra pared rectal } \\
\text { (corresponde al } 25 \% \text { de los nódulos } \\
\text { retroperitoneales) }\end{array}$ \\
\hline
\end{tabular}

rante el estudio de pacientes con algia pelviana crónica (cíclica o no cíclica), no muestra utilidad en el diagnóstico de endometriosis del tabique rectovaginal o intestinal. Su utilidad se restringe a aquellos casos en que coexisten endometriomas $(25,27)$. Su capacidad diagnóstica con los endometriomas justifica su uso ya que la endometriosis profunda con compromiso intestinal rara vez se presenta en forma aislada y generalmente coexiste con estas otras formas de endometriosis pelviana $(9,14,23)$.

En nuestra serie, en uno de los casos coexistían endometriomas y endometriosis profunda con compromiso colorrectal ( 1 de 3 casos). Aunque nuestra serie es pequeña, tal frecuencia de coexistencia concuerda con lo reportado en la literatura. Esto es, entre 39,7 a $53 \%$ de los casos de endometriosis profunda coexisten con endometriomas $(26,28)$. Cabe agregar además, que la presencia de endometriomas es un marcador de enfermedad severa y un factor de riesgo independiente para la necesi-

Tabla III

EFICACIA DE LOS DIFERENTES EXÁMENES (VALORES PROMEDIO) EN EL DIAGNÓSTICO DE COMPROMISO INTESTINAL EN PACIENTES CON ENDOMETRIOSIS PROFUNDA DE LA PELVIS POSTERIOR

\begin{tabular}{|c|c|c|c|c|c|c|c|}
\hline Examen & $\begin{array}{l}\text { Sensibilidad } \\
(\%)\end{array}$ & $\begin{array}{c}\text { Especificidad } \\
(\%)\end{array}$ & $\begin{array}{l}\text { VPP } \\
(\%)\end{array}$ & $\begin{array}{l}\text { VPN } \\
(\%)\end{array}$ & $\begin{array}{l}\mathrm{LR} \\
(+)^{*}\end{array}$ & $\begin{array}{l}\mathrm{LR} \\
(-)^{*}\end{array}$ & 'Gold Standard' \\
\hline USTR (24) & 100 & 98 & 75 & 100 & 50 & - & Cirugía, histología \\
\hline USTV $(25,26)$ & 91 & 98 & 97 & 91 & 46 & 0,1 & Cirugía, histología \\
\hline $\begin{array}{l}\text { Endosonografía } \\
(25,28,33,37)\end{array}$ & 92 & 84 & 85 & 89 & 5,8 & 0,1 & Cirugía, histología \\
\hline $\begin{array}{l}\text { RM de pelvis }(28 \\
30,35,37)\end{array}$ & 78 & 93 & 90 & 83 & 11 & 0,2 & Cirugía, histología \\
\hline $\begin{array}{l}\text { Enema baritado } \\
\text { (32) }\end{array}$ & 100 & 98 & 98 & 100 & 50 & - & Cirugía, histología \\
\hline $\begin{array}{l}\text { TAC- enteroclisis } \\
\text { (38) }\end{array}$ & 99 & 100 & 100 & 96 & - & 0,01 & Histología \\
\hline
\end{tabular}

* Valores calculados en base a sensibilidad y especificidad entregados por los autores.

USTR ultrasonografía transrectal

USTV ultrasonografía transvaginal

RNM resonancia nuclear magnética

TAC tomografía axial computarizada

ble (25). Bazot y cols (26), en un estudio prospectivo controlado, en el que se incluyeron 142 pacientes, demostraron que la USTV era una herramienta útil en el diagnóstico del compromiso endometriósico intestinal. Sin embargo, al igual que con la USTR, requería que el radiólogo estuviera familiarizado tanto con el aspecto normal de la pared colorrectal como cuando se encuentra comprometido por estas lesiones. En nuestra experiencia, aunque la USTV constituye el examen de primera línea du-
VPP valor predictivo positivo VPN valor predictivo negativo LR Likelihood ratio

dad de resección intestinal durante el tratamiento quirúrgico. En un análisis de 1785 pacientes con endometriosis ovárica, sólo el $1 \%$ tenía endometriomas como lesión única (29). Esto significa que, si un ginecólogo diagnostica y trata solo la endometriosis ovárica, existe un $99 \%$ de posibilidad de dejar sin tratamiento otras lesiones mayores (29). Por ello el ginecólogo, ante el hallazgo de endometriomas, debe esperar enfrentarse a una enfermedad extensa y a la posibilidad de terminar en una cirugía 
compleja con el riesgo de complicaciones mayores. Aunque los estudios de diagnóstico no lo sugieren, durante la exploración quirúrgica debe buscarse siempre otras lesiones y de haberlas, tratarlas.

La RM de pelvis constituye una herramienta diagnóstica cada vez más utilizada en la evaluación preoperatoria de la endometriosis profunda de la pelvis posterior. Esta técnica es superior a la ultrasonografía, pues no es operador dependiente y entrega información más completa y objetiva de la anatomía de la pelvis. La RM es muy precisa en el diagnóstico en ciertas localizaciones de la endometriosis profunda como el tabique rectovaginal o el rectosigmoides. Sin embargo, la RM muestra algunas limitaciones con la presencia de endometriomas en la cercanía de los ligamentos uterosacros o con úteros en retroversión (30). En nuestra experiencia, la RM tiene gran correlación con los hallazgos intraoperatorios.

Un examen que ha entrado en desuso pero que ha sido utilizado por más de 30 años, ante la sospecha de compromiso intestinal, es el enema baritado de doble contraste (31). Al respecto sólo un estudio ha evaluado su eficacia diagnóstica (32). En ese examen es posible observar la deformación de la pared anterior del colon o rectosigmoides por la presencia de la enfermedad. En nuestro centro no lo utilizamos de forma rutinaria.

La endosonografía constituye actualmente el examen de elección ante la sospecha de compromiso endometriósico de la pared intestinal (9). Este examen permite delimitar con gran exactitud tanto la profundidad de la lesión que afecta a la pared intestinal como la distancia de ella al margen anal (33-36). Chapron y cols (37), demostraron que la endosonografía tiene una mayor sensibilidad y valor predictivo negativo que la RM para el diagnóstico de compromiso rectal en pacientes con endometriosis profunda de la pelvis posterior. Sin embargo, este examen tiene desventajas. Dentro de ellas está el requerimiento de un operador entrenado, la incomodidad de una preparación previa mediante enema evacuante y la visualización de sólo una pequeña porción de la pelvis. En nuestro centro la hemos utilizado sólo en aquellos casos en que existe una fuerte sospecha clínica de compromiso rectal.

Recientemente, ha surgido evidencia con el uso de la tomografía axial computarizada (TAC) multicorte con enteroclisis. Este examen pareciera ser un método muy eficaz en el diagnóstico de endometriosis intestinal $(38,39)$. A la fecha, no contamos con experiencia con esta técnica en pacientes con endometriosis.

Un examen frecuentemente realizado en pacientes con sospecha de endometriosis profunda con compromiso intestinal es la colonoscopia. Con ella se busca identificar lesiones con compromiso transmural intestinal y poder biopsiarlas. Sin embargo, el compromiso de la mucosa es extremadamente infrecuente, por lo que habitualmente el examen resultará normal o con indicios de compresión extrínseca. De observar lesiones y biopsiarlas, ellas mostrarán sólo inflamación crónica lo cual puede confundir o generar interpretaciones erróneas $(6,40)$, tal como ocurrió en uno de nuestros casos en donde el diagnóstico planteado osciló entre un cuadro de fibrosis retroperitoneal, sospecha de patología neoplásica y endometriosis profunda. En nuestra opinión, el mayor rol de la colonoscopía estaría en el realizar un diagnóstico diferencial de las pacientes con rectorragia y en excluir de tumores malignos.

Confirmado el diagnóstico de endometriosis profunda con compromiso intestinal, la única alternativa terapéutica razonable y efectiva, en pacientes sintomáticas, es lograr la resección quirúrgica completa del tejido endometrial ectópico. Sin embargo, tal cirugía es extremadamente compleja y se asocia a posibles complicaciones intestinales, ureterales y vaginales (41). Por ende, la planificación previa y la coordinación en la participación de un equipo multidisciplinario (ginecólogo, urólogo y cirujano digestivo) es crucial.

Respecto al uso de terapia médica, antes o después de la cirugía, ya sea con el fin de facilitar el procedimiento o para disminuir el riesgo de recurrencia, no existe evidencia que lo avale (42). Pese a ello, en algunos casos, puede constituir una opción previa a la cirugía (difiriendo el momento de ésta), particularmente si la alternativa quirúrgica implica una cirugía demasiado compleja y/o riesgosa o si se trata de paciente de alto riesgo quirúrgico (ej. pacientes con trastornos de la coagulación) $(9,43)$. Cabe señalar, que tal opción es sólo transitoria y no debe constituir la alternativa de manejo definitivo. Tanto en nuestra experiencia como en la de otros grupos, la eficacia del tratamiento médico en pacientes con endometriosis profunda es mínima con una tasa alta de recurrencias sintomáticas, requiriendo en definitiva, de cirugía en la mayoría de los casos $(10,11,44)$.

Un aspecto a considerar en las pacientes con esta enfermedad se refiere al compromiso por enfermedad de otras estructuras pelvianas, particularmente el uréter. Dicho compromiso es variable en extensión y profundidad. Habitualmente corresponde a un atrapamiento o estenosis secundaria a la fibrosis circundando al nódulo rectovaginal y que lo compromete por vecindad. Afortunadamente tal compromiso es infrecuente. Sin embargo, en 
aquellos nódulos de gran tamaño $(\geq 3 \mathrm{~cm})$ y/o con extensión lateral, tal compromiso puede alcanzar el $11 \%$ de los casos (4). El establecer su diagnóstico previo o durante la cirugía es de vital importancia pues, sin tratamiento y producto de la obstrucción, puede llevar a la pérdida silente e irreversible de la función renal (4). Síntomas sugerentes de uropatía obstructiva o la ocurrencia de lumbalgia catamenial son poco frecuentes y la mayoría de las pacientes permanecen asintomáticas (45). Por ende, en nódulos de gran tamaño y/o con extensión lateral siempre debe estudiarse dirigidamente la vía urinaria independiente de que existan o no síntomas asociados. La pielografía de eliminación es el examen de elección frente a la presunción de compromiso ureteral (22). Cabe señalar que nuestras dos pacientes con compromiso ureteral no presentaban síntomas urinarios. Basado en lo anterior, es que hemos decidido en nuestro centro el realizar estudio mediante pielografía, como parte de la evaluación preoperatoria, a todas las pacientes con nódulo tipo III según la clasificación de Donnez (Tabla II), los mayores de $3 \mathrm{~cm}$ o aquellos con extensión lateral. En estas pacientes, la instalación preoperatorio de catéteres doble $\mathrm{J}$ permite la identificación del uréter y facilita la ureterolisis. Aún así, el tratamiento quirúrgico es complejo y puede requerir de la resección parcial del uréter y el posterior reimplante de la porción proximal en la vejiga. Se han descrito diferentes técnicas de reimplante ureteral, siendo la vejiga psoica y el Casati-Boari las más utilizadas (45). En nuestra serie, uno de los casos requirió de una vejiga psoica. En el segundo caso, producto del compromiso extenso y bilateral, la solución fue el reimplante ureteral mediante un doble CasatiBoari (en escopeta de cañón yuxtapuesto).

Frecuentemente la endometriosis intestinal es un hallazgo intraoperatorio inesperado para el ginecólogo, el cual con frecuencia no cuenta con un entrenamiento adecuado en cirugía intestinal. Lo anterior, sumado a la falta de una adecuada preparación intestinal, determinan muchas veces que el procedimiento termine en una cirugía incompleta para esta entidad. Debido a esto, es que recomendamos realizar siempre preparación intestinal ante la sospecha o hallazgo de endometriomas como también en toda paciente con antecedente de endometriosis. A ello se agrega el trabajo coordinado con un cirujano digestivo quien ante el hallazgo pueda concurrir a asistir en su manejo.

La técnica y abordaje quirúrgico escogidos dependerán tanto de la extensión y localización de la enfermedad como de la experiencia del cirujano. No existe consenso claro sobre las indicaciones con las cuales debe realizarse una resección intes- tinal en endometriosis, pero en general se indica en pacientes sintomáticas, cuando existe sospecha de malignidad, ante la presencia de implantes profundos que comprometan $>50 \%$ del perímetro intestinal, con nódulos $>3 \mathrm{~cm}$, múltiples nódulos o con compromiso transmural $(9,46,47,48)$. Las candidatas a esta cirugía deberán ser adecuadamente informadas sobre la posibilidad de conversión, de lesión ureteral, de colostomía y de la persistencia de síntomas urinarios y digestivos pos cirugía.

Durante años tanto el compromiso profundo de la pared intestinal como la necesidad de reimplante ureteral constituyeron indicación absoluta de laparotomía $(23,49)$. La Tabla IV resume los resultados de las principales series de endometriosis profunda con compromiso intestinal en las cuales se realizó resección intestinal segmentaria por laparotomía. Sin embargo, el escenario ha cambiado, puesto que los avances en la técnica laparoscópica y la mayor experiencia de los cirujanos con ella, han permitido abordar esta patología por esta vía.

Desde la realización de la primera resección intestinal laparoscópica por endometriosis en 1991 (53), ésta opción se ha transformado en una alternativa posible y cada vez más atractiva para el manejo de la endometriosis profunda de la pelvis posterior con compromiso intestinal (54). El abordaje mínimamente invasivo ofrece resultados comparables a la cirugía abierta, teniendo la ventaja de minimizar el trauma quirúrgico y permitir una mejor visualización de la pelvis profunda $(49,55,56)$. Sin embargo, tal forma de abordaje requiere de cirujanos expertos y bien entrenados. No basta contar con un ginecólogo laparoscopista sino que el resto del equipo debe manejarse con dichas técnicas (cirujano digestivo y urólogo).

La Tabla $V$ resume los resultados de las principales series de resección intestinal en endometriosis profunda realizada por laparoscopia.

Debe enfatizarse con algunas de estas series que la resección segmentaria constituye una pequeña proporción del total de casos con cirugía intestinal por endometriosis pelviana $(47,57,58)$.

Es importante recalcar que la conversión a laparotomía no debe considerarse una complicación, sino como un escenario posible en el contexto de una cirugía de gran complejidad. Tanto en nuestra serie como en otras $(57,63)$, la mayoría de las conversiones ocurrieron en pacientes que requirieron de una resección segmentaria o reimplante ureteral. Diferente es el caso con otras formas de endometriosis donde la conversión es infrecuente al tratarse de cirugías menos complejas.

Al analizar los resultados de series con pacientes operadas por endometriosis profunda con com- 


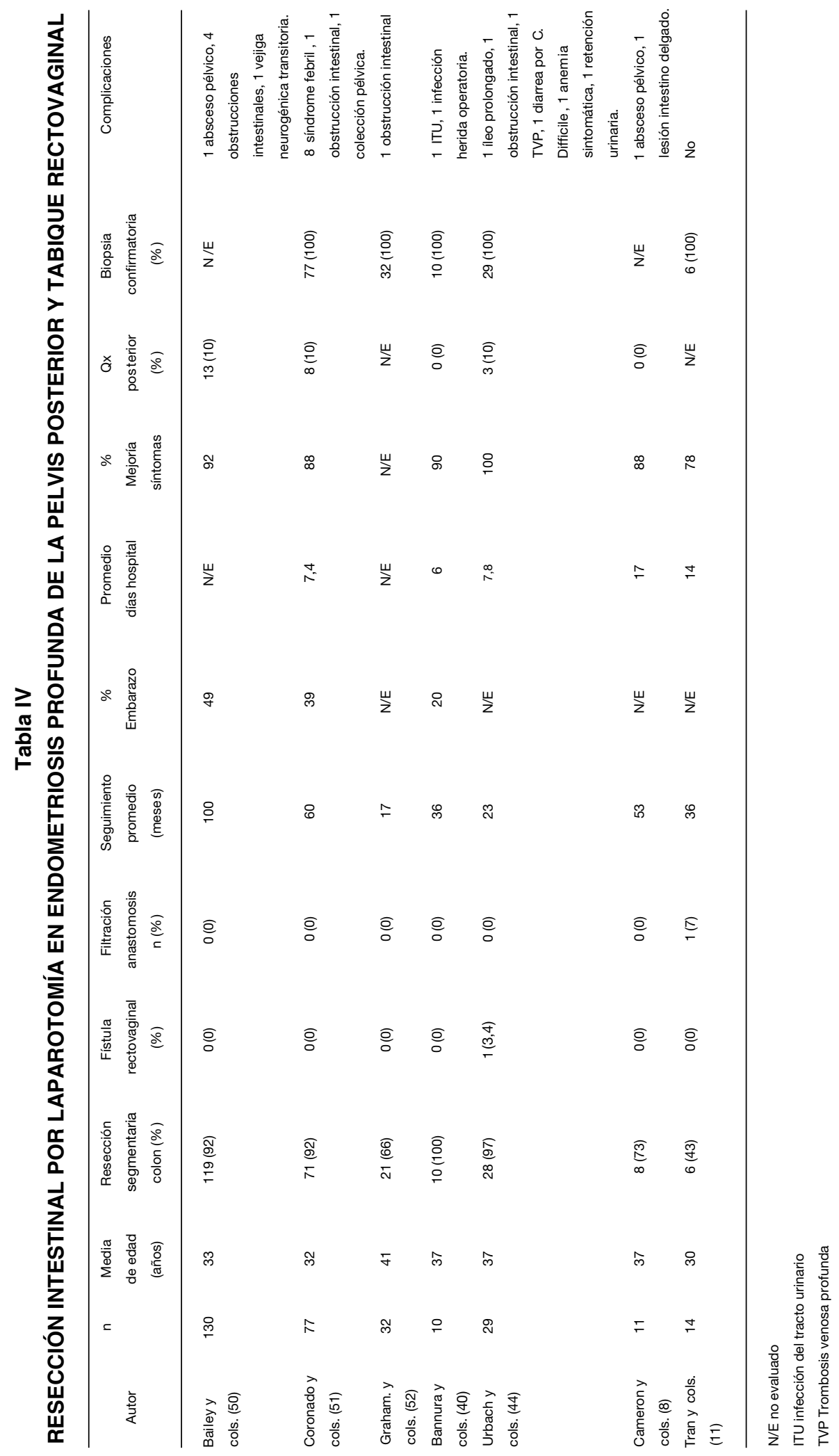




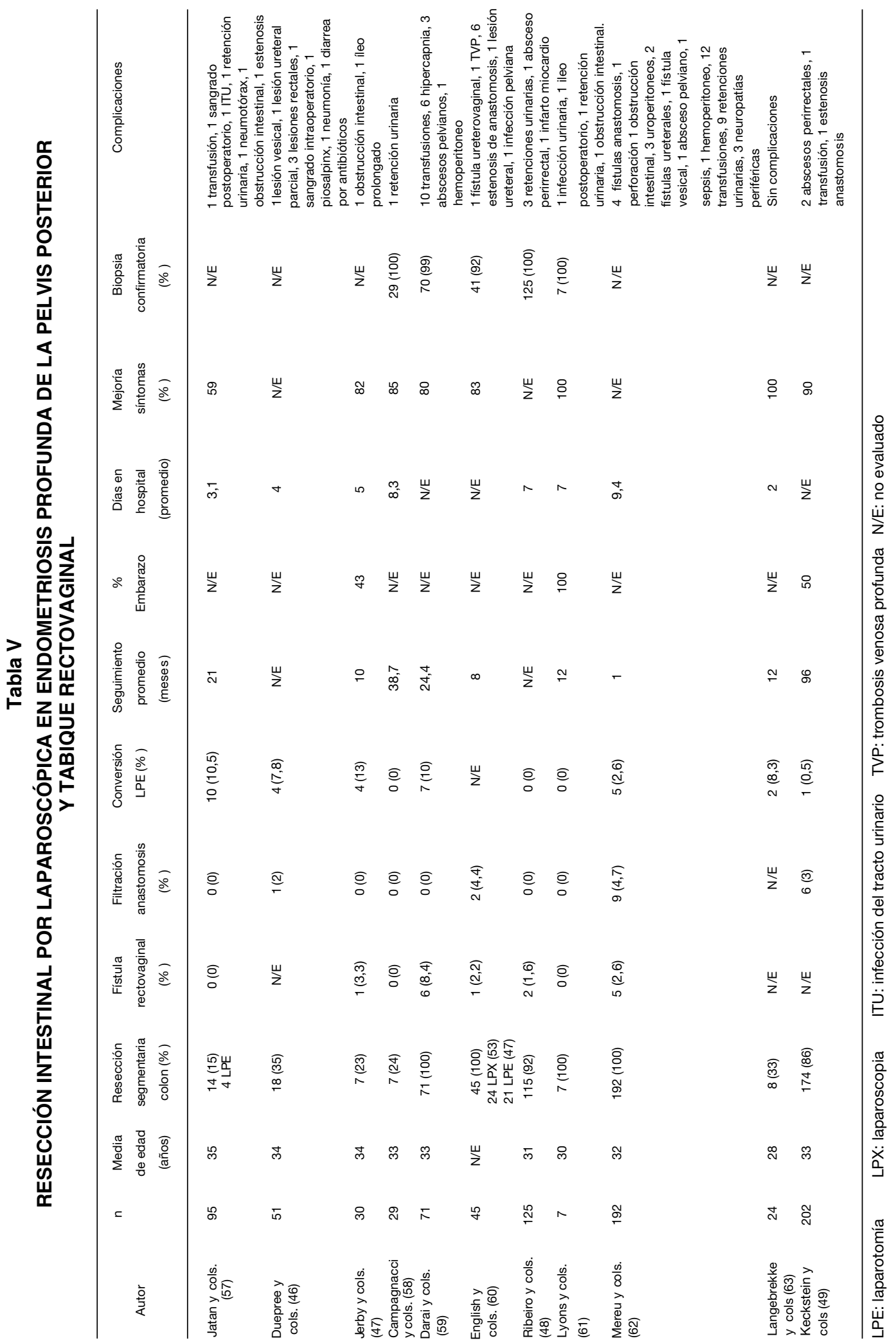


promiso intestinal, en que fue necesario realizar una resección intestinal, podemos señalar que son buenos, con tasas de morbilidad aceptables y resultados satisfactorios respecto a fertilidad y mejoría en la calidad de vida $(56,64)$. Nuestra casuística es aún pequeña para sacar conclusiones sólidas pero al menos avala la factibilidad y seguridad del procedimiento, posterior a un estudio y cirugía bien planificada.

\section{CONCLUSIÓN}

La endometriosis profunda con compromiso intestinal corresponde a una variedad infrecuente dentro de las distintas formas de la endometriosis. Pese a ello, el ginecólogo no puede estar exento de ofrecer un adecuado manejo. Esta entidad abre una nueva frontera y una fuente de desafío para el especialista, obligando al conocimiento más avanzado de la anatomía y de una nueva fisiopatología. Junto a ello, hace necesario que a su entrenamiento concurran conocimientos y práctica que tradicionalmente han sido considerados propios de otras áreas. Además de un alto índice de sospecha, clave en el diagnóstico, es necesario realizar un estudio adecuado donde la RM de pelvis y la endosonografía tienen un rol central. Finalmente, es necesaria una adecuada planificación del tratamiento, el que puede requerir de cirugías de gran complejidad. El tratamiento quirúrgico, ya sea por laparoscopia o laparotomía, es efectivo en disminuir la sintomatología, siempre y cuando se logre la remoción completa de la enfermedad. Por último, el tratamiento quirúrgico debe ser individualizado y realizarse en centros especializados donde el manejo multidisciplinario sea realmente posible.

Agradecimientos: Los autores agradecen al Sr. Hernán Ahumada Poblete y a la Sra. Carmen Gloria Sandoval Tillería, asistentes de biblioteca de la Pontificia Universidad Católica de Chile, por su asistencia en la recolección del material utilizado en la elaboración de este manuscrito.

\section{BIBLIOGRAFÍA}

1. Giudice LC, Kao LC. Endometriosis. Lancet 2004;364:1789-99.

2. Cornillie FJ, Oosterlynck D, Lauweryns JM, Koninckx PR. Deeply infiltrating pelvis endometriosis: histology and clinical significance. Fertil Steril 1990;53:978-83.

3. Martin DC, Batt RE. Retrocervical, rectovaginal pouch, and rectovaginal septum endometriosis. J Am Assoc Gynecol Laparosc 2001;8:12-7.

4. Donnez J, Nisolle M, Squifflet J. Ureteral endometriosis: a complication of rectovaginal endometriotic (adenomyotic) nodules. Fertil Steril 2002;77:32-7.

5. Weed JC, Ray JE. Endometriosis of the bowel. Obstet Gynecol 1987;69:727-30.
6. Zwas FR, Lyon DT. Endometriosis. An important condition in clinical gastroenterology. Dig Dis Sci 1991;36:35364.

7. Vercellini P, Chapron C, Fedele L, Gattei U, Daguati $R$, Crosignani $P G$. Evidence for asymmetric distribution of lower intestinal tract endometriosis. BJOG 2004;111:1213-17.

8. Cameron IC, Rogers S, Collins MC, Reed MW. Intestinal endometriosis: presentation, investigation, and surgical management. Int J Colorect Dis 1995;10:836.

9. Remorgida V, Ferrero S, Fulcheri E, Ragni N, Martin D. Bowel endometriosis: Presentation, diagnosis, and treatment. Obstet Gynecol Surv 2007;62:461-70.

10. Waller KG, Shaw RW. Gonadotropin-releasing hormone analogues for the treatment of endometriosis: long-term follow-up. Fertil Steril 1993;59:511-15.

11. Tran KT, Kuijpers HC, Willemsen WN, Bulten H. Surgical treatment of symptomatic rectosigmoid endometriosis. Eur J Surg 1996;162:139-41.

12. Cuello M. Profilaxis perioperatoria. En: Cuello $M$, Pomés $C$ (eds). Pautas de práctica clínica en ginecología. Santiago: Ediciones Universidad Católica de Chile 2005;21-3.

13. Sampson JA. Intestinal adenomas of endometrial type. Arch Surg 1922;5:217-21.

14. Chapron C, Fauconnier A, Vieira M, Barakat H, Dousset $\mathrm{B}$, Pansini $\mathrm{V}$, et al. Anatomical distribution of deeply infiltrating endometriosis: surgical implications and proposition for a classification. Hum Reprod 2003;18:157-61.

15. Adamson DG. Diagnosis and clinical presentation of endometriosis. Am J Obstet Gynecol 1990;162:5689.

16. Chapron $\mathrm{C}$, Barakat $\mathrm{H}$, Fritel $\mathrm{X}$, Dubuisson JB, Bréart G, Fauconnier A. Presurgical diagnosis of posterior deep infiltrating endometriosis based on a standardized questionnaire. Hum Reprod 2005;20:507-13.

17. Fauconnier A, Chapron C, Dubuisson JB, Vieira M, Dousset B, Bréart $\mathrm{G}$. Relation between pain symptoms and the anatomic location of deep infiltrating endometriosis. Fertil Steril 2002;78:719-26.

18. Koninckx PR, Meuleman C, Oosterlynck D, Cornillie FJ. Diagnosis of deep endometriosis by clinical examination during menstruación and plasma CA-125 concentration. Fertil Steril 1996;65:280-7.

19. Chapron C, Dubuisson JB, Pansini V, Vieira M, Fauconnier A, Barakat $\mathrm{H}$, et al. Routine clinical examination is not sufficient for diagnosing and locating deeply infiltrating endometriosis. J Am Assoc Gynecol Laparosc 2002;9:115-9.

20. Dragisic KG, Padilla LA, Milad MP. The accuracy of the rectovaginal examination in detecting cul-de-sac disease in patients under general anaesthesia. Hum Reprod 2003;18:1712-5.

21. Adamyan L. Additional international perspectives. En: Nichols DH (ed). Gynecologic and Obstetric Surgery. St Louis: Mosby-Year Book 1993;1167-82.

22. Squifflet J, Feger C, Donnez J. Diagnosis and imaging of adenomyotic disease of the retroperitoneal space. Gynecol Obstet Invest 2002;54(suppl 1):43-51.

23. Chapron C, Chopin N, Borghese B, Malartic C, Decuypere F, Foulot $H$. Surgical management of deeply 
infiltrating endometriosis: an update. Ann NY Acad Sci 2004;1034:326-37.

24. Fedele L, Bianchi S, Portuese A, Borruto F, Dorta M. Transrectal ultrasonography in the assessment of rectovaginal endometriosis. Obstet Gynecol 1998;91:4448.

25. Bazot M, Detchev R, Cortez A, Amouyal P, Uzan S, Darai E. Transvaginal sonography and rectal endoscopic sonography for the assessment of pelvic endometriosis: a preliminary comparison. Hum Reprod 2003;18:1686-92.

26. Bazot M, Thomassin I, Hourani R, Cortez A, Darai E. Diagnostic accuracy of transvaginal sonography for deep pelvic endometriosis. Ultrasound Obstet Gynecol 2004;24:180-5.

27. Kinkel K, Frei KA, Balleyguier C, Chapron C. Diagnosis of endometriosis with imaging: a review. Eur Radiol 2006;16:285-98.

28. Bazot M, Bornier C, Dubernard G, Roseau G, Cortez A, Darai E. Accuracy of magnetic resonance imaging and rectal endoscopic sonography for the prediction of location of deep pelvic endometriosis. Hum Reprod 2007;22:1457-63.

29. Redwine DB. Ovarian endometriosis: a marker for more extensive pelvis and intestinal disease. Fertil Steril 1999;72:310-5.

30. Bazot M, Darai E, Hourani R, Thomassin I, Cortez A, Uzan S, et al. Deep pelvic endometriosis: MR imaging for diagnosis and prediction of extension of disease. Radiology 2004;232:379-89.

31. Gordon RL, Evers K, Kressel HY, Laufer I, Herlinger $\mathrm{H}$, Thompson JJ. Double-contrast enema in pelvic endometriosis. AJR Am J Roentgenol 1982;138:549-52.

32. Landi S, Barbieri F, Fiaccavento A, Mainardi P, Ruffo G, Selvaggi $\mathrm{L}$, et al. Preoperative double-contrast barium enema in patients with suspected intestinal endometriosis. J Am Assoc Gynecol Laparosc 2004;11:223-8.

33. Delpy R, Barthet M, Gasmi M, Berdah S, Shojai R, Desjeux $A$, et al. Value of endorectal ultrasonography for diagnosing rectovaginal septal endometriosis infiltrating the rectum. Endoscopy 2005;37:357-61.

34. Bahr A, de Parades V, Gadonneix P, Etienney I, SaletLizée $\mathrm{D}$, Villet $\mathrm{R}$, et al. Endorectal ultrasonography in predicting rectal wall infiltration in patients with deep pelvic endometriosis: a modern tool for an ancient disease. Dis Colon Rectum 2006;49:869-75.

35. Camagna O, Dhainaut $C$, Dupuis $O$, Soncini E, Martin $\mathrm{B}$, Palazzo L, et al. Prise en charge chirugicale des endométrioses de la cloison rectovaginale. À propos d’une série continue de 50 cas. [Surgical management of rectovaginal septum endometriosis from a continuous series of 50 cases]. Gynecol Obstet Fertil 2004;32:199-209.

36 Doniec JM, Kahlke V, Peetz F, Schniewind B, Mundhenke C, Löhnert MS, et al. Rectal endometriosis: high sensitivity and specificity of endorectal ultrasound with an impact for the operative management. Dis CoIon Rectum 2003;46:1667-73.

37. Chapron C, Vieira M, Chopin N, Balleyguier C, Barakat $\mathrm{H}$, Dumontier I, et al. Accuracy of rectal endoscopic ultrasonography and magnetic resonance imaging in the diagnosis of rectal involvement for patients presenting with deeply infiltrating endometriosis. Ultrasound Obstet Gynecol 2004;24:175-9.

38. Biscaldi E, Ferrero S, Fulcheri E, Ragni N, Remorgida V, Rollandi GA. Multislice CT enteroclysis in the diagnosis of bowel endometriosis. Eur Radiol 2007; 17: 211-9.

39. Biscaldi E, Ferrero S, Remorgida V, Rollandi GA. Bowel endometriosis: CT-enteroclysis. Abdom Imaging 2007;32(4):441-50.

40. Bannura G, Melo C, Contreras J, Barrera A, Soto D, Liendo $\mathrm{R}$, et al. Resultados del tratamiento quirúrgico de la endometriosis colorrectal: Análisis de 10 pacientes consecutivas. Rev Chil Cir 2004;56:117-24.

41. Donnez J, Pirard C, Smets M, Jadoul P, Squifflet J. Surgical management of endometriosis. Best Pract Res Clin Obstet Gynaecol 2004;18:329-48.

42. Yap C, Furness S, Farquhar C. Pre and post operative medical therapy for endometriosis surgery. Cochrane Database Syst Rev 2004;3:CD003678.

43. Martin DC. Rectovaginal endometriosis. J Obstet Gynecol Ind 2000;50:178-81.

44. Urbach DR, Reedijk M, Richard CS, Lie KI, Ross TM. Bowel resection for intestinal endometriosis. Dis CoIon Rectum 1998;41:1158-64.

45. Antonelli A, Simeone C, Frego E, Minini G, Bianchi $\mathrm{U}$, Cunico SC. Surgical treatment of ureteral obstruction from endometriosis: our experience with thirteen cases. Int Urogynecol J Pelvic Floor Dysfunct 2004;15:407-12.

46. Duepree HJ, Senagore AJ, Delaney CP, Marcello PW, Brady KM, Falcone T. Laparoscopic resection of deep pelvic endometriosis with rectosigmoid involvement. J Am Coll Surg 2002;195:754-8.

47. Jerby BL, Kessler H, Falcone T, Milsom JW. Laparoscopic management of colorectal endometriosis. Surg Endosc 1999;13:1125-8.

48. Ribeiro PA, Rodrigues FC, Kehdi IP, Rossini L, Abdalla HS, Donadio N, et al. Laparoscopic resection of intestinal endometriosis: a 5-year experience. J Minim Invasive Gynecol 2006;13:442-6.

49. Keckstein J, Wiesinger H. Deep endometriosis, including intestinal involvement--the interdisciplinary approach. Minim Invasive Ther Allied Technol 2005;14:1606.

50. Bailey HR, Ott MT, Hartendorp P. Aggressive surgical management for advanced colorectal endometriosis. Dis Colon Rectum 1994;37:747-53.

51. Coronado C, Franklin RR, Lotze EC, Bailey HR, Valdés CT. Surgical treatment of symptomatic colorectal endometriosis. Fertil Steril 1990;53:411-6.

52. Graham B, Mazier WP. Diagnosis and management of endometriosis of the colon and rectum. Dis Colon Rectum 1988;31:952-6.

53. Redwine DB, Sharpe DR. Laparoscopic segmental resection of the sigmoid colon for endometriosis. J Laparoendosc Surg 1991;1:217-20.

54. Remorgida V, Ragni N, Ferrero S, Anserini P, ToreIli $P$, Fulcheri $E$. How complete is full thickness disc resection of bowel endometriotic lesions? A prospective surgical and histological study. Hum Reprod 2005;20:2317-20.

55. Wright JT, Redwine DB. Treatment of endometriosis- 
a special skills module only? Gynecol Surg 2004;1:678.

56. Ret Dávalos ML, De Cicco C, D'Hoore A, De Decker B, Koninckx PR. Outcome after rectum or sigmoid resection: a review for gynecologists. J Minim Invasive Gynecol 2007;14:33-8.

57. Jatan AK, Solomon MJ, Young J, Cooper M, PathmaNathan N. Laparoscopic management of rectal endometriosis. Dis Colon Rectum 2006;49:169-74.

58. Campagnacci R, Perretta $S$, Guerrieri M, Paganini AM, De Sanctis A, Ciavattini A, et al. Laparoscopic colorectal resection for endometriosis. Surg Endosc 2005; 19:662-4.

59. Darai E, Ackerman G, Bazot M, Rouzier R, Dubernard G. Laparoscopic segmental colorectal resection for endometriosis: limits and complications. Surg Endosc 2007;21:1572-7.

60. English J, Kenney N, Edmonds S, Baig MK, Miles A. Limited segmental anterior resection for the treatment of rectovaginal endometriosis: pain and complications. Gynecol Surg 2007;4:107-10.
61. Lyons SD, Chew SS, Thomson AJ, Lenart M, Camaris C, Vancaillie TG, et al. Clinical and quality-of-life outcomes after fertility-sparing laparoscopic surgery with bowel resection for severe endometriosis. J Minim Invasive Gynecol 2006;13:436-41.

62. Mereu L, Ruffo G, Landi S, Barbieri F, Zaccoletti $\mathrm{R}$, Fiaccavento $A$, et al. Laparoscopic treatment of deep endometriosis with segmental colorectal resection: short-term morbidity. J Minim Invasive Gynecol 2007; 14:463-9.

63. Langebrekke A, Istre $O$, Busund $B$, Johannessen $\mathrm{HO}$, Qvigstad E. Endoscopic treatment of deep infiltrating endometriosis (DIE) involving the bladder and rectosigmoid colon. Acta Obstet Gynecol Scand 2006;85:712-5.

64. Daraï E, Bazot M, Rouzier R, Houry S, Dubernard G. Outcome of laparoscopic colorectal resection for endometriosis. Curr Opin Obstet Gynecol 2007;19:30813. 